\title{
Simulation and Fabrication of the Cone Sheet for LCD Backlight Application
}

\author{
Sang Hoon Baik, Sung Ki Hwang, Young Gyu Kim, Gyeungju Park, and Jin Hyuk Kwon* \\ Department of Physics, Yeungnam University, Gyeongsan 712-749, Korea \\ Won Taek Moon, Sung Hoon Kim, Byoung Ku Kim, and Sin-Ho Kang \\ LG Display, Paju 730-726, Korea
}

(Received September 4, 2009 : revised November 9, 2009 : accepted November 13, 2009)

\begin{abstract}
An optical sheet with a cone array is designed, simulated, and fabricated in order to substitute the dual crossed prism sheets in the edge-type LCD backlight. The optimum structure of cone textures that is compatible with the dual crossed prism sheets was obtained by simulating the backlight installed with the cone array optical sheet. A SU-8 photoresist films of thickness 30 50 $\mu \mathrm{m}$ were spin-coated on a polyethylene terephtalate film (PET), and the cone texture array was formed by using the diffuse lithography that employed a photomask with circular patterns and an optical diffuser.
\end{abstract}

Keywords: Backlight, Prism sheet, Cone texture

OCIS codes : (120.2040) Displays; (120.4610) Optical fabrication; (120.5240) Photometry

\section{INTRODUCTION}

A recent trend of the liquid crystal display (LCD) backlight is to develop a slim, cheap, and energy efficient backlight with fewer optical films [1-2]. Especially, notebook LCDs require high normal luminance with relatively small view angle compared to the LCD TVs that require a large view angle. As shown in Fig. 1, an edgetype backlight consists of a light source placed at an

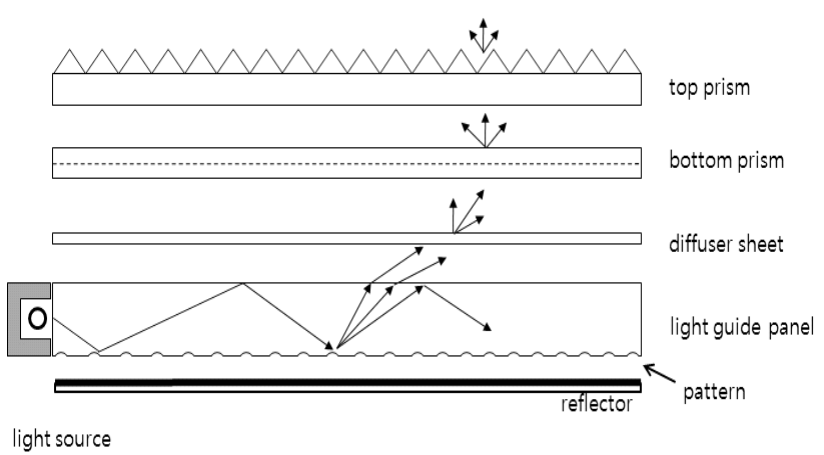

FIG. 1. A conventional structure of an edge-lit LCD backlight unit with dual crossed prism sheets. edge of the lightguide panel, a diffuser sheet, a bottom prism sheet, a top prism sheet [3]. The dual crossed prism sheets that consist of the bottom and the top prism sheets in crossed directions are used to enhance the normal luminance in notebook LCDs. It is necessary to develop a cost-effective optical sheet that can compete with the dual crossed prism sheets because the dual crossed prism sheets are the most expensive optical components in the backlight unit [4-5]. The dual prism sheets placed in crossed directions generate a high normal luminance and a narrow view angle as small as about 50 degrees. So, a new optical sheet that can replace the dual prism sheets with compatible performance needs to be developed. One of the candidates is the microlens array optical sheet and the other is the cone array sheet. The microlens array optical sheet with apertures on the reverse side has the normal luminance and the view angle compatible to the dual crossed prism sheets [6-7]. In this study, the feasibility of a cone array sheet with apertures on the reverse side to replace the dual crossed prism sheets was studied by simulation and the possibility of making the cone texture by using diffuse lithography was studied [7-8].

*Corresponding author: jhkwon@ynu.ac.kr 


\section{SIMULATION OF THE CONE ARRAY SHEET}

Fig. 2 shows the design parameters of the cone texture. These are the top radius $R_{1}$, the bottom radius $\mathrm{R}_{2}$, the cone height $\mathrm{H}$, and the pitch between adjacent cones. Other parameters that affect the backlight performance are the pitch distance, spacing, and the layout geometry of the cone textures. In this study, the spacing between the adjacent cone textures was set at $10 \mu \mathrm{m}$, which is the minimum distance to separate real cone textures. The layout of the cone textures was assumed to be triangular, which maximizes the density of cone textures per unit area. A simulation model of the edgetype backlight as shown in Fig. 3 was constructed by using the Light Tools Simulation Program. The dimension of the backlight simulation model was 13.5 " in the diagonal direction with a lightguide panel of thickness $0.7 \mathrm{~mm}$. A CCFL with length $274.3 \mathrm{~mm}$ and inner diameter $0.5 \mathrm{~mm}$ was placed along the longer edge of the lightguide. The bottom surface of the lightguide panel was assumed to have concave microlens array textures with Bezier distribution having a weighting factor 0.41 to give uniform illuminance distribution at the upper surface of the lightguide. The diameter and height of the microlenses were $0.1 \mathrm{~mm}$ and $0.025 \mathrm{~mm}$, respectively. A Gaussian reflecting film with spread value 15 degrees

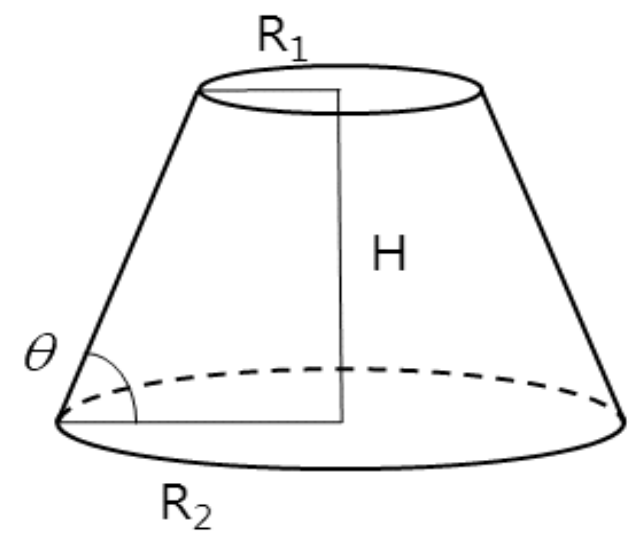

FIG. 2. Design parameters of a cone texture. $\mathrm{R}_{1}$; top radius, $\mathrm{R}_{2}$; bottom radius, $\mathrm{H}$; height, $\theta$; side angle.

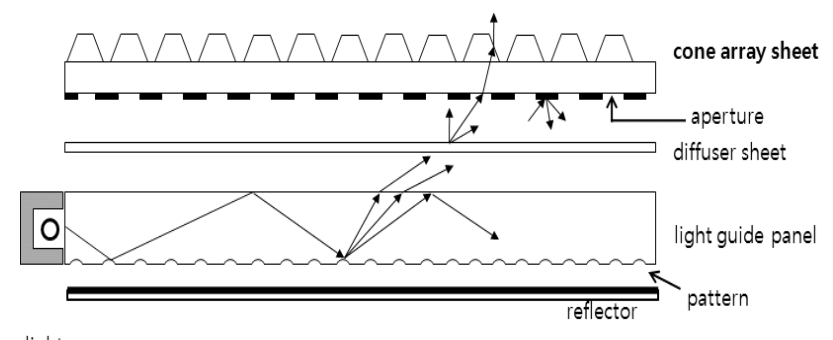

light source

FIG. 3. A schematic structure of LCD backlight unit with the cone array sheet replacing the dual crossed prism sheets. and reflectivity $98 \%$ through the visible range were assumed to be beneath the lightguide panel. The diffuser sheet placed between the lightguide panel and the prism sheet was assumed to have the Gaussian scattering characteristics with a spread value of 15 degrees and transmittance of $90 \%$. The cone array sheet was assumed to be made of apolyethylene terephtalate (PET) that has the refractive index 1.575 at $550 \mathrm{~nm}$.

Fig. 4 shows the simulation results of the angular luminance profiles of the dual crossed prism sheets which were placed on top of the diffuser sheet. The normal luminance was $5,656 \mathrm{~cd} / \mathrm{m}^{2}$ and the view angle is about 52.5 degrees and these values were used as the reference of comparison to the cone array sheet. Because two prism sheets are stacked in crossed directions, the view angle is decreased but the normal luminance is increased. Due to the narrow view angle and high normal luminance, the dual crossed prism sheets are used in most notebook backlights because less electrical energy is consumed. The strong sidelights at the angle of 80 degrees from the

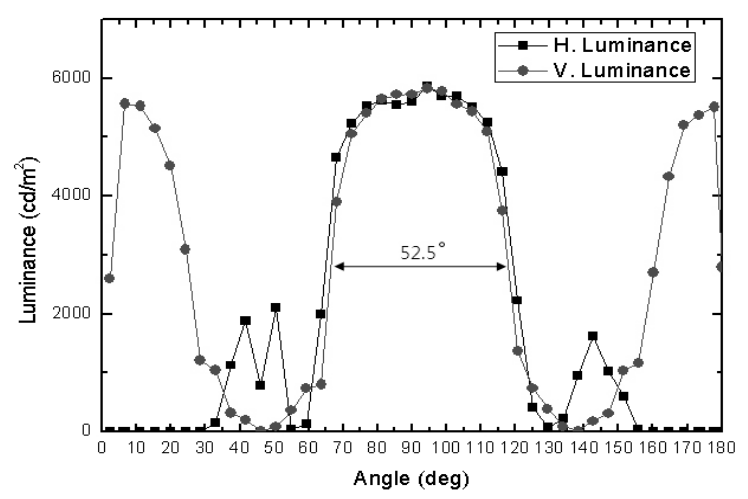

FIG. 4. The simulation results of the horizontal and vertical angular luminance profiles of the dual crossed prism sheets in Cartesian coordinates.

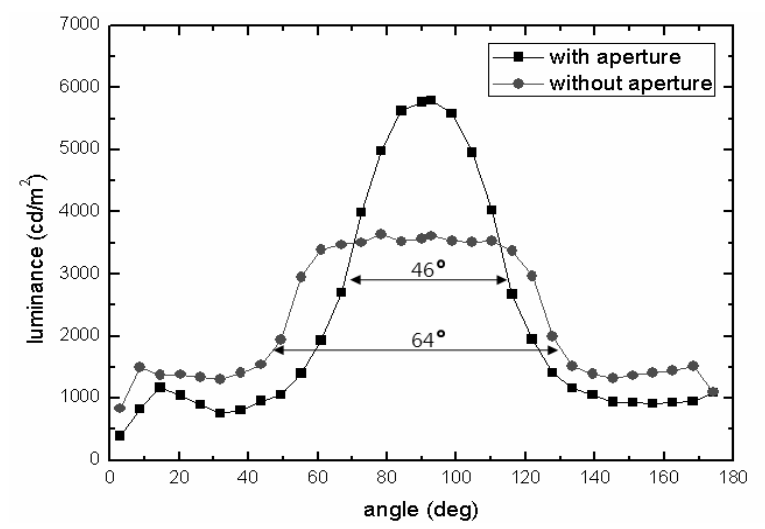

FIG. 5. The simulation results of the horizontal and vertical angular luminance profiles of cone sheets with and without apertures on the reverse surface of the cone array sheet. The view angle is given by the full-width at half maximum (FWHM). 
normal direction shown in Fig. 4 degrade the contrast ratio and so these sidelights should be minimized if possible.

Fig. 5 shows a typical simulation result of the angular luminance profiles of the cone array sheet with and without apertures on the reverse side. The simulation parameters were top radius $R_{1}=10 \mu \mathrm{m}$, lower radius $R_{2}=50 \mu \mathrm{m}$, cone height $\mathrm{H}=40 \mu \mathrm{m}$, cone side angle $\theta=45$ degrees, the reflectivity of the reverse surface $\mathrm{R}=90 \%$, the diameter of the aperture $\mathrm{D}=50 \mu \mathrm{m}$, and substrate thickness $\mathrm{T}=75 \mu \mathrm{m}$. As shown in Fig. 5, when there are apertures on the reverse surface the normal luminance and the view angle were about $102.4 \%$ of dual crossed prism sheet and 46 degrees, respectively. However, when there are no apertures as shown in Fig. 5, the normal luminance was only $62.9 \%$ of the dual crossed prism sheets and the view angle was about 64 degrees. These simulation results show that the normal luminance and view angle can be improved a lot by applying a reflective layer to the reverse surface of the film with transparent apertures at corresponding positions of the cone textures. The reflecting surface of the reverse surface was assumed to have the Gaussian scattering characteristics with spread value of 15 degrees.

Fig. 6 shows the change of the normal luminance and the view angle as reflectance of the opposite surface was increased from $75 \%$ to $95 \%$ in steps of $5 \%$. The thickness, the aperture diameter and the side angle of the reverse surface of the cone array sheet were assumed to be $75 \mu \mathrm{m}, 50 \mu \mathrm{m}$, and 45 degree, respectively. The normal luminance was increased from $87.3 \%$ to 107.6 $\%$, and the view angle decreased from 63 degrees to 57.6 degrees with negligible sidelights. When the reflectivity is higher than $90 \%$, the normal luminance becomes larger than that of the dual crossed prism sheets.

Fig. 7 shows the normal luminance and view angle as a function of the aperture diameter for the reverse surface reflectivity of $\mathrm{R}=80 \%$ and $90 \%$. The thickness, the reflectivity of reverse surface, and side angle were

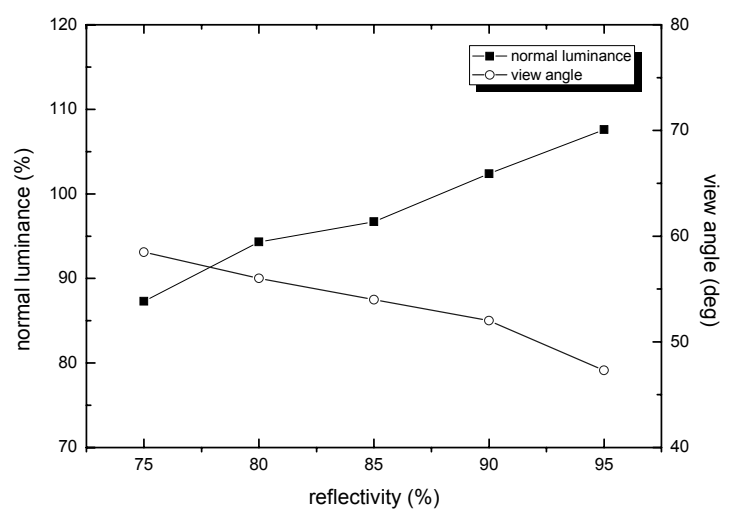

FIG. 6. The change of normal luminance and view angle as the reflectivity of the reverse surface is increased. The normal luminance is given by percentage of the normal luminance of the dual prism sheets. assumed to be $75 \mu \mathrm{m}, 90 \%$, and 45 degree. The normal luminance is given by percentage of the normal luminance of the dual prism sheets for comparison. When the aperture diameter is $50 \mu \mathrm{m}$ and the reverse surface reflectivity is $90 \%$, the maximum normal luminance is $102.4 \%$ of the dual crossed prism sheets. The view angle is increased from 38 degree to 67.5 degree as the aperture diameter is increased from $20 \mu \mathrm{m}$ to $90 \mu \mathrm{m}$. However, the when the reflectivity of the reverse surface is given as $80 \%$, the maximum normal luminance is decreased to $94.3 \%$ and the view angle shows a minimum value at the aperture diameter of $50 \mathrm{um}$. This is because the stray light through the reflective surface tends to enlarge the view angle.

Fig. 8 shows the relative change of the normal luminance in terms of the side angle of the cone texture. When the side angle was changed from 45 degrees to 70 degrees, the normal luminance decreased from about

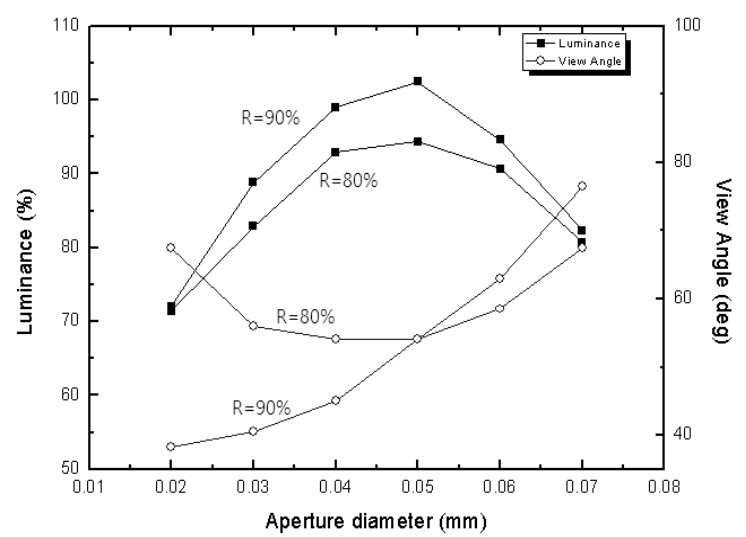

FIG. 7. The normal luminance and view angle as a function of the aperture diameter for the revese surface reflectivity of $\mathrm{R}=80 \%$ and $90 \%$. The normal luminance is given by percentage of the normal luminance of the dual prism sheets.

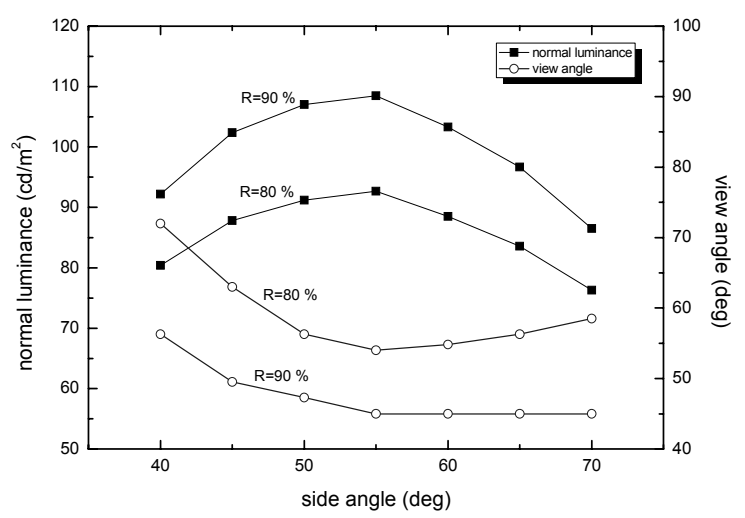

FIG. 8. The change of the normal luminance in terms of the side angle of the cone texture. The unit of normal luminance is given by percentage for comparison to the normal luminance of the dual crossed prism sheets. 
$88.2 \%$ to $67.6 \%$ compared to the dual crossed prism sheets. The thickness, the aperture diameter, and the reflectivity of the reverse surface was assumed to be 75 $\mu \mathrm{m}, 50 \mu \mathrm{m}$, and $90 \%$, respectively. The maximum normal luminance reaches as high as $108.5 \%$ of the normal luminance of the dual crossed prism sheets but the view angle is decreased to 45 degrees. When the side angle is 45 degrees, the normal luminance is $102.4 \%$ and the view angle is about 49.5 degrees, which are comparable values with those of the dual crossed prism sheets. Fig. 9 shows the change of the normal luminance and the view angle as a function of the substrate thickness of the cone array sheet for the simulation parameters of the side angle 45 degree, aperture diameter $50 \mu \mathrm{m}$, the reflectivity of the reverse surface $\mathrm{R}=90 \%$. It is seen that the normal luminance has a peak value at the substrate thickness $90 \mu \mathrm{m}$ but the view angle in FWHM is decreased to 42.8 degrees. When the thickness is given as $75 \mu \mathrm{m}$, the normal luminance and the view angle become $102.4 \%$ and $94 \%$ of the dual crossed prism sheets. The view angle goes under 35 degrees and the angular luminance profiles begin to show strong side lights if the substrate thickness is over $120 \mu \mathrm{m}$. The narrow view angle comes from the collimating property of the cone texture because the apertures are located at the rough focal points of the cone textures. So, the optimum thickness of the cone array sheet with apertures can be determined within $60 \sim 100 \mu \mathrm{m}$ according to the needed view angle.

The simulation results showed that the normal luminance of the cone array sheets with apertures ranges from $71.4 \%$ to $108.5 \%$ of the normal luminance of the dual crossed prism sheets and the view angle is increased from 35 degrees to about 58 63 degrees according to the aperture diameter, the substrate thickness, and the reflectivity of the reverse surface. The optimum design parameters of the cone textures that show compatible characteristics with the dual

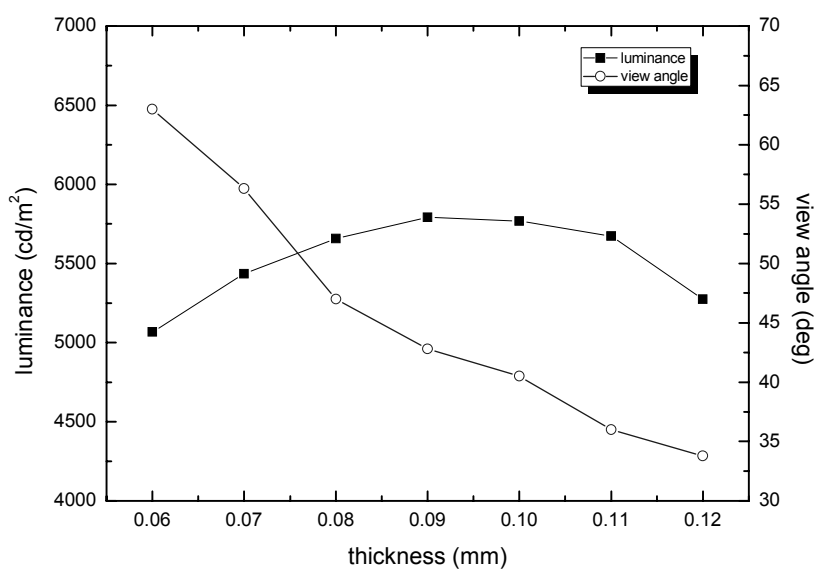

FIG. 9. The change of the normal luminance and the view angle as a function of the thickness of the cone sheet. crossed prism sheets will be the aperture size $50 \mu \mathrm{m}$ for the bottom radius $100 \mu \mathrm{m}$, the thickness $60 \sim 100$ $\mu \mathrm{m}$, and the reflectivity of the reverse surface $90 \%$.

\section{FABRICATION OF CONE ARRAY SHEET BY USING THE DIFFUSE LITHOGRAPHY}

The cone array textures were made by the diffuse lithographic method [9]. The photolithographic process to fabricate cone array sheet consists of 6 steps; spin coating, soft baking, exposure, post exposure baking (PEB), developing, and rinsing. First, we designed and made a photomask with circular patterns with specifications of the circle diameter $=20 \mu \mathrm{m}$ and the pitch $=80$ $\mu \mathrm{m}$. The circular patterns were placed in triangular positions. The substrates were PET films of about 100 $\mu \mathrm{m}$ in thickness. A PET film of size $100 \mathrm{~mm} \times 100 \mathrm{~mm}$ was placed on the chuck of a spin coater and the photoresist SU-8 that is transparent in the visible range was spin-coated to make a photoresist film of thickness $50 \mu \mathrm{m}$. A two-step soft baking was performed during 10 minutes at $65^{\circ} \mathrm{C}$ and 30 minutes at $95^{\circ} \mathrm{C}$ on a hot plate in order to remove the volatile agents completely [10]. The exposure was performed during 10 minutes by ultraviolet in a setup for the diffuse lithography that is comprised of an opal diffuser, a photomask, and a PET film substrate as shown in Fig. 10. The PEB was performed on a hot plate in two steps; the first baking at $65^{\circ} \mathrm{C}$ and the second baking at $95^{\circ} \mathrm{C}$. The cone array sheet was completed after

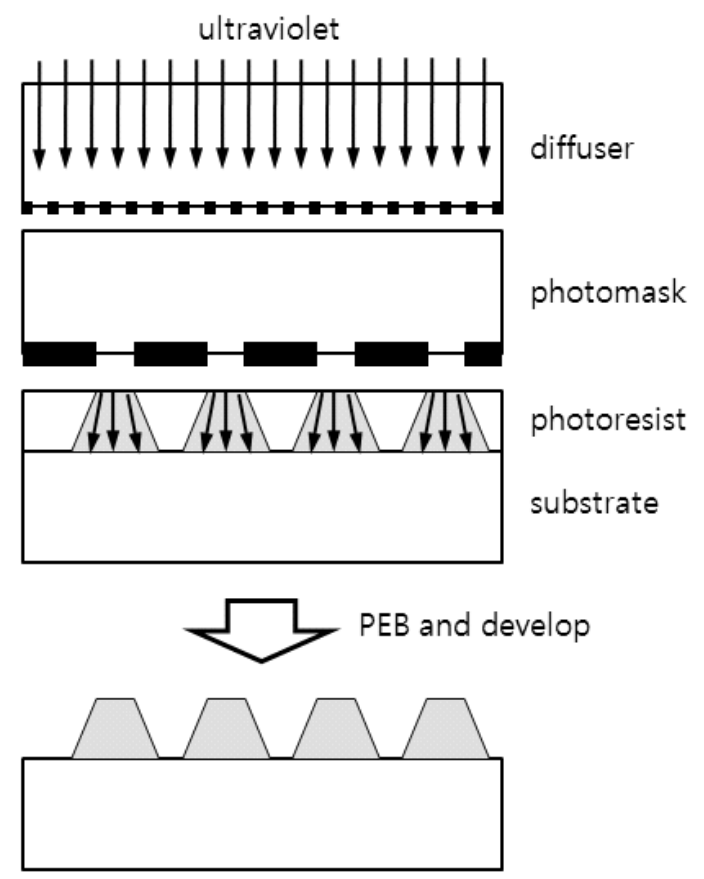

FIG. 10. The exposure schematic for fabricating cone textures by the diffuse lithography. 
developing in a SU-8 developer, rinsing in an isopropyl alcohol, and drying in air.

The fabricated cone textures were investigated by using a microscope, a scanning electron microscope (SEM), and a white light interferometer that can analyze the three dimensional structures precisely. The white light interferometer measures two-dimensional and threedimensional profiles by using white light interference fringes that have coherence length as small as a few micrometers [11]. Fig. 11 shows a SEM picture of the cone texture array fabricated by the diffuse lithography setup as shown in Fig. 10, and Fig. 12 shows the 3 dimensional profiles of the cone textures obtained from the white light interferometer [12].

The side angle $\theta$ of the cone was calculated from the top radius $R_{1}$, the bottom radius $R_{2}$, and the height

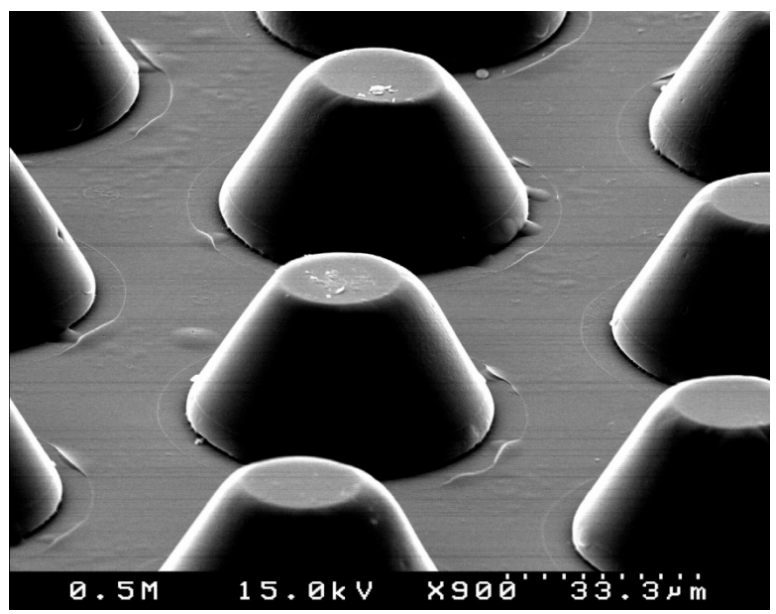

FIG. 11. A SEM picture of the cone textures fabricated by diffuse lithography.

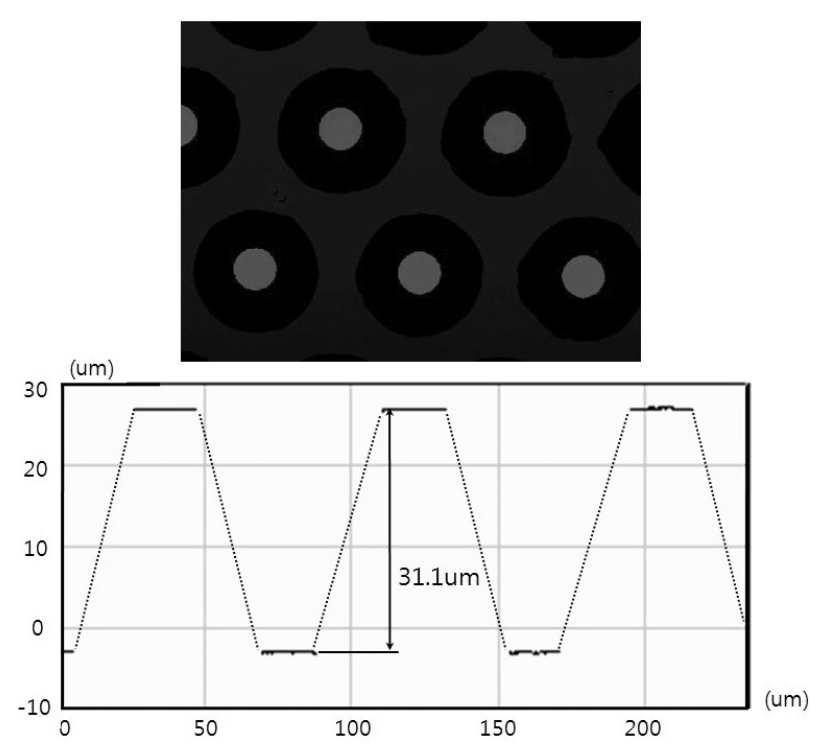

FIG. 12. The three dimensional profile of cone textures measured by the white light interferometer.

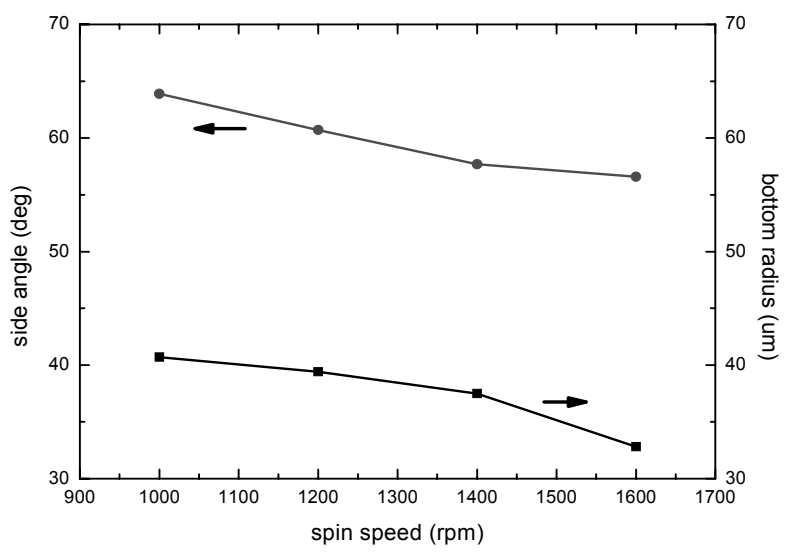

FIG. 13. The change of side angle and bottom radius of the cone textures in terms of the spin speed of the spin coater. The top radius was $10 \mu \mathrm{m}$, which is the same as the photomask circular pattern, and the photoresist was SU-8.

of cone $\mathrm{H}$ by the following equation;

$$
\theta=\tan ^{-1}\left(\frac{H}{R_{2}-R_{1}}\right)
$$

When the spin speed was 1,000rpm, the measured values of $R_{2}$ and $H$ obtained from the white light interferometer were $40.7 \mu \mathrm{m}$ and $62.7 \mu \mathrm{m}$, respectively, and the calculated side angle was $\theta=63.9$ degrees. Fig. 13 shows the change of the side angle and the bottom radius of the cone textures in terms of the spin speed. The top radius was $10 \mu \mathrm{m}$, the same as that of the photomask circular pattern. It is seen that the side angles can be controlled between 56 degrees and 64 degrees in the diffuse lithography.

\section{CONCLUSION}

The simulation results show that the angular luminance of the cone sheet without apertures on the reverse side is only $62.9 \%$ of that of the dual crossed prism sheet, but the angular luminance of the cone sheet with apertures goes as high as about $108.5 \%$ of that of the dual crossed prism sheet, so it is possible for a single cone array sheet with apertures to replace the dual crossed prism sheets. Also, we have shown that the cone texture array can be fabricated by using the diffuse lithography. The side angle was controlled between 56 degrees and 64 degrees. The remaining task is to establish how to mass-produce the cone textures on a film and to fabricate the apertures on the reflecting layer of the substrate. The apertures and the reflective layer placed on the reverse side of the substrate can 
be fabricated by using resins that include scattering beads or by applying a reflective paste by the screen printing technology. The screen printing combined with the roll-process seems to be adequate for the mass production of the cone sheet with apertures.

\section{ACKNOWLEDGMENT}

This work was partially supported by the Regional Innovation Center Program (ADMRC) of the Ministry of Knowledge Economy, Republic of Korea.

\section{REFERENCES}

1. M. Anandan, "LCD backlighting," SID '01 Seminar Lecture Notes F-2/1 (2001).

2. I. K. Kim and K. Y. Chung, "Wide color gamut backlight from three-band white LED," J. Opt. Soc. Korea 11, 67-70 (2007).

3. D. H. Lee, "Optimization design and analysis of LCD backlight unit,” M.S. Thesis, Yeungnam University (2006), pp. 11-13.

4. J. H. Ko, "Recent research trends in the development of new light source for the backlight unit of liquid crystal display," Asian J. Phys. 14, 231-237 (2005).
5. G. Kurata, K. Sakurai, M. Ohira, Y. Kawabata, A. Funamoto, and A. Aoyama, "Sheetless backlight system for mobile phone," in Proc. IDW'06 (Otsu, Japan, Dec. 2006), pp. 1727-1730.

6. T. Susumu, "Liquid crystal display apparatus," Japanese Patent 2000-284268 (2000).

7. Y. Hira, “Optical film," U.S. Patent 6,639,705 (2003).

8. Y. H. Ju, J. H. Park, J. H. Lee, J. Y. Lee, K. B. Nahm, and J. H. Ko, "Study on the simulation model for the optimization of optical structures of edge-lit backlight for LCD applications," J. Opt. Soc. Korea 12, 25-30 (2008).

9. J. H. Lee, H. S. Lee, B. K. Lee, W. S. Choi, H. Y. Choi, and J. B. Yoon, "Simple liquid crystal display backlight unit comprising only a single-sheet micropatterned polydimethylsiloxane (PDMS) light-guide plate," Opt. Lett. 32, 2665-2667 (2007).

10. M. Brunet, T. O’Donnell, J. O’Brien, P. McCloskey, and S. C. Mathuna, "Thick photoresist development for the fabrication of high aspect ratio magnetic coils," J. Micromech. Microeng. 12, 444-449 (2002).

11. Y. J. Chuang, F. G. Tseng, J. H. Cheng, and W. K. Lin, "A novel fabrication method of embedded microchannels by using SU-8 thick-film photoresists," Sens. Actuators A 103, 64-69 (2003).

12. P. J. Caber, "Three-dimensional imaging by sub-Nyquist sampling of white-light interferograms," Opt. Lett. 18, 1462-1464 (1993). 